\title{
Genetic Analysis in Pearl Millet [Pennisetum glaucum (L) R. Br.]
}

\author{
M.R. Reshma Krishnan ${ }^{1 *}$, M.S. Patel ${ }^{2}$, R.A. Gami ${ }^{3}$, H.S. Bhadauria ${ }^{1}$ and Y.N. Patel ${ }^{2}$ \\ ${ }^{1}$ Department of Genetics and Plant Breeding, CPCA, \\ S.D. Agricultural University, Sardarkrushinagar, Gujarat, India \\ ${ }^{2}$ Center for Crop Improvement, S.D. Agricultural University, Sardarkrushinagar, Gujarat, India \\ ${ }^{3}$ Maize Research Station, S.D. Agricultural University, Bhiloda-383 245, Gujarat, India \\ *Corresponding author
}

\section{Keywords}

Pear millet, $\mathrm{L} x \mathrm{~T}$ design, combining ability, GCA, SCA Heterosis.

Article Info

Accepted:

10 September 2017

Available Online:

10 November 2017

\section{A B S T R A C T}

The present investigation was undertaken with the objectives to assess the magnitude of heterosis and combining ability effects of 35 hybrids resulted from Line $\mathrm{x}$ Tester mating design involving seven lines and five testers parents along with the standard check (GHB 558). The highly significant analysis of variance for parents and hybrids among all traits revealed that, the existence of appreciable amount of genetic variability in the experimental material. The analysis of variance for combining ability revealed that specific combining ability variance for $\mathrm{m} x \mathrm{f}$ interaction were highly significant for all characters. The magnitude of sca variances was higher than the gca variances for all the characters. This indicated non-additive gene action in the inheritance of these traits. The female ICMA 96222 had high per se performance and good general combing ability for days to flowering, days to maturity, ear head length, test weight, grain yield per plant, harvest index and protein content. The cross ICMA 06777 x $18805 \mathrm{R}$ had good $\mathrm{x}$ average combiner parents, high per se performance, significant positive heterobeltiosis and standard heterosis and positive specific combining ability effect for grain yield per plant, test weight, ear head length and harvest index. The cross ICMA 96222 x $18488 \mathrm{R}$ had good x good combiner parent, significant positive specific combining effect for grain yield per plant test weight, ear head length and protein content.

\section{Introduction}

Pearl millet [Pennisetum glaucum (L.) R. Br.] (also known under synonyms: $P$. americanum (L.) Leeke or P. typhoides (Burm.) Stapf and C.E. Hubb.) is an important millet crop of traditional farming systems in tropical and subtropical Asia and sub-Saharan Africa. Discovery of $\mathrm{A}_{1}$ cytoplasmic-nuclear male sterility (CGMS) at Tifton, Georgia, USA (Burton 1958) initiated the era of hybrid cultivar development in pearl millet [Pennisetum glaucum (L). R. Br.], which led to the release of the first grain hybrid in India in 1965 (Athwal, 1965). Since then hundreds of commercial hybrids, all of them based on the $\mathrm{A}_{1}$-CMS system, have been developed and released or commercialized. This dependence on single cytoplasm makes pearl millet hybrid seed industry vulnerable to disease and insectpest epidemics. This concern compelled the search for new sources of CMS in pearl millet (Rai et al., 2006). Hanna (1989) identified an $\mathrm{A}_{4}$ CMS system at Tifton, Georgia, USA in a 
wild grassy Pennisetum glaucum (L.) R. Br. subsp. monodii (Maire) Brunken. The combining ability studies provide useful information regarding the selection of suitable parents for effective hybridization programme and at the same time elucidates the nature and magnitude of gene action. In order to proceed with any breeding programme, the breeder has to know about the gene action and the genetic architecture of population. Since, the nature of gene action varies with genetic architecture of population involved in hybridization, it is necessary to evaluate the parents for their combining ability. Evaluation of crosses and identification of the superior cross combinations (combining ability) for yield and quality traits essential for effective manipulation through hybrid breeding.

\section{Materials and Methods}

The experimental material consisted of seven maintainer lines used as female lines (ICMA 06777, ICMA -07777, ICMA-96222, ICMA97111, ICMA-98444, ICMA-04999 and ICMA-05444) and five restorer lines used as testers (J18488-R, 18587-R, 18805-R, 17369$\mathrm{R}$ and 17548-R), crossed in a Line $\mathrm{x}$ Tester mating design. The resultant 35 hybrids along with their 12 parents with check GHB-558 were evaluated in Randomized Block Design with three replications at Centre for Crop Improvement, S. D. Agricultural University, Sardarkrushinagar-385 506 (Gujarat) during summer 2016. The observations were recorded on five randomly selected competitive plants of each genotype in each replication for various characters i.e. days to flowering, days to maturity, plant height $(\mathrm{cm})$, number of effective tillers per plant, ear head length, ear head girth, test weight, grain yield per plant, harvest index and protein content. Days to flowering (DF) on the basis of $50 \%$ plants of each genotype flowered, days to maturity (DM) on the basis of $80 \%$ plants of each genotype matured were recorded. The protein content (PC) was estimated in percentage by using NIR spectroscopy technique. The replication wise mean values were used in statistical analysis. The data were subjected to analysis of variance as per the procedure suggested by Sukhatme and Amble (1989). The combining ability analysis was performed for a Line $\mathrm{x}$ Tester matting design as per the method suggested by Kempthorne (1957). The hybrid performance (\%) tested in comparison with mean value of two parents (Relative heterosis/RH), better parent (heterobeltiosis/BPH) and standard check (Standard heterosis/SH) suggested by Briggle (1963), Fonseca and Patterson (1968) and Meredith and Bridge (1972) respectively.

\section{Results and Discussion}

The analysis of variance depicting mean squares due to genotypes were highly significant for all the characters are presented in Table 1. Further, partitioning of the genotypes variance into parents, hybrids and parents vs. hybrids revealed that the parents as well as hybrids exhibited significant differences for all the traits.

This indicated the existence of appreciable amount of genetic variability in the experimental material. The comparison of parents vs. hybrids were highly significant for all the traits except protein content indicating that the presence of heterosis.

The mean squares obtained in analysis of variance for combining ability and estimated components of genetic variance for various characters are presented in Table 2 . The result revealed that the mean squares due to females were highly significant for days to flowering, days to maturity, ear head length, ear head girth, test weight and harvest index. This indicated significant contribution of females towards general combining ability variance component for these traits. 
Table.1 Analysis of variance (Mean square) for parents and hybrids for seed yield and its component characters in pearl millet

\begin{tabular}{|c|c|c|c|c|c|c|c|c|c|c|c|}
\hline Source of variation & d.f & $\begin{array}{c}\text { Days to } \\
\text { flowering }\end{array}$ & $\begin{array}{l}\text { Days to } \\
\text { maturity }\end{array}$ & $\begin{array}{c}\text { Plant height } \\
(\mathrm{cm})\end{array}$ & $\begin{array}{c}\text { No. of } \\
\text { effective } \\
\text { tillers per } \\
\text { plant }\end{array}$ & $\begin{array}{c}\text { Earhead } \\
\text { length } \\
(\mathrm{cm})\end{array}$ & $\begin{array}{l}\text { Earhead } \\
\operatorname{girth}(\mathrm{mm})\end{array}$ & $\begin{array}{c}\text { Test } \\
\text { weight } \\
\text { (g) }\end{array}$ & $\begin{array}{c}\text { Grain yield } \\
\text { per plant } \\
(\mathrm{g})\end{array}$ & $\begin{array}{c}\text { Harvest } \\
\text { index } \\
(\%)\end{array}$ & $\begin{array}{c}\text { Protein } \\
\text { content } \\
(\%)\end{array}$ \\
\hline Replication & 2 & 3.13 & 1.68 & 866.36 & 0.03 & 1.65 & 3.51 & 0.35 & 9.53 & 2.89 & 0.70 \\
\hline Genotype & 46 & $71.08 * *$ & $86.15^{* *}$ & $5163.65 * *$ & $1.00 * *$ & $38.68 * *$ & $72.66^{* *}$ & $5.10^{* *}$ & $46.63 * *$ & $169.69 * *$ & $3.36 * *$ \\
\hline Parents & 11 & $73.48 * *$ & $80.13 * *$ & $6504.91 * *$ & $0.91^{* *}$ & $26.50 * *$ & $61.00 * *$ & $6.32 * *$ & $15.07 * *$ & $170.07 * *$ & $3.91 * *$ \\
\hline Female & 6 & $50.54 * *$ & $96.08 * *$ & $891.96^{*}$ & $0.40 * *$ & $26.79 * *$ & $83.30 * *$ & $7.44 * *$ & $21.47 * *$ & $125.04 * *$ & $3.22 * *$ \\
\hline Male & 4 & $57.43 * *$ & $53.43 * *$ & 217.37 & $1.89 * *$ & $32.08 * *$ & $42.56 * *$ & $5.38 * *$ & 8.95 & $226.64 * *$ & $4.93 * *$ \\
\hline Female vs. male & 1 & $275.33 * *$ & $91.21 * *$ & $65332.80 * *$ & 0.03 & 2.47 & 0.96 & $3.39 * *$ & 1.11 & $213.99 * *$ & $3.96 * *$ \\
\hline Parents vs. hybrids & 1 & $41.46 * *$ & $124.47 * *$ & $41908.36 * *$ & $0.45^{* *}$ & $133.51 * *$ & $283.43 * *$ & $1.75^{* *}$ & $123.42 * *$ & $39.97 *$ & 0.31 \\
\hline hybrids & 34 & $71.17 * *$ & $86.97 * *$ & $3648.98 * *$ & $1.05 * *$ & $39.83 * *$ & $70.23 * *$ & $4.81 * *$ & $54.59 * *$ & $173.38 * *$ & $3.27 * *$ \\
\hline Error & 92 & 1.54 & $0.96 * *$ & 333.65 & 0.02 & 2.87 & 2.30 & 0.16 & 6.15 & 7.74 & 0.25 \\
\hline
\end{tabular}

$*$ and $* *$ indicates significant at $\mathrm{P}=0.05$ and $\mathrm{P}=0.01$ levels respectively.

Table.2 Analysis of variance (Mean square) for combining ability, and estimates of components of variance for various characters in pearl millet

\begin{tabular}{|c|c|c|c|c|c|c|c|c|c|c|c|}
\hline $\begin{array}{c}\text { Source of } \\
\text { variation }\end{array}$ & d.f & $\begin{array}{c}\text { Days to } \\
\text { flowering }\end{array}$ & $\begin{array}{c}\text { Days to } \\
\text { maturity }\end{array}$ & Plant height & $\begin{array}{l}\text { No. of effective } \\
\text { tillers per plant }\end{array}$ & $\begin{array}{c}\text { Ear head } \\
\text { length }\end{array}$ & $\begin{array}{c}\text { Ear head } \\
\text { girth }\end{array}$ & $\begin{array}{c}\text { Test } \\
\text { weight }\end{array}$ & $\begin{array}{c}\text { Grain yield } \\
\text { per plant }\end{array}$ & $\begin{array}{c}\text { Harvest } \\
\text { index }\end{array}$ & $\begin{array}{l}\text { Protein } \\
\text { content }\end{array}$ \\
\hline Replication & 2 & 4.06 & 2.6 & 602.52 & $0.11 *$ & 2.183 & 3.64 & 0.317 & 6.45 & 4.16 & 0.48 \\
\hline Crosses & 34 & $71.17 * *$ & $86.97 * *$ & $3648.98 * *$ & $1.04 * *$ & $39.82 * *$ & $70.23 * *$ & $4.80 * *$ & $54.59 * *$ & $173.37 * *$ & $3.27 * *$ \\
\hline Females (Line) & 6 & $195.68 * *$ & $308.08 * *$ & 4683.52 & 1.41 & $50.66 *$ & $207.16^{* *}$ & $11.10 * *$ & 71.45 & $650.57 * *$ & 4.34 \\
\hline Males (Tester) & 4 & 48.62 & $91.73 *$ & 7414.11 & 0.42 & $148.16 * *$ & $115.80 *$ & $8.93 *$ & 58.07 & 44.41 & 2.63 \\
\hline Females $\times$ males & 24 & $43.80 * *$ & $30.89 * *$ & $2762.827 * *$ & $1.06 * *$ & $19.05 * *$ & $28.40 * *$ & $2.54 * *$ & $49.79 * *$ & $75.57 * *$ & $3.10 * *$ \\
\hline Error & 68 & 1.43 & 0.99 & 440.62 & 0.02 & 2.67 & 2.99 & 0.18 & 5.89 & 9.19 & 0.211 \\
\hline \multicolumn{2}{|l|}{$\boldsymbol{\sigma}^{2}$ Females } & $10.12 * *$ & $18.47 * *$ & 128.05 & 0.02 & $2.10 *$ & $11.91 * *$ & $0.57 * *$ & 1.44 & $38.33 * *$ & 0.08 \\
\hline \multicolumn{2}{|l|}{$\boldsymbol{\sigma}^{2}$ Males } & 0.22 & $2.89 *$ & 221.49 & -0.03 & $6.14 * *$ & $4.16 *$ & $0.30 *$ & 0.39 & -1.48 & -0.02 \\
\hline \multicolumn{2}{|l|}{$\sigma^{2}$ gca } & $4.35 * *$ & $9.38 * *$ & $182.55 * *$ & 0.01 & $4.46 * *$ & $7.39 * *$ & $0.41 * *$ & $0.83 *$ & $15.10 * *$ & 0.02 \\
\hline \multicolumn{2}{|l|}{$\sigma^{2}$ sca } & $14.08 * *$ & $9.97 * *$ & $809.72 * *$ & $0.34 * *$ & $5.39 * *$ & $8.70 * *$ & $0.79 * *$ & $14.54 * *$ & $22.60 * *$ & $0.95 * *$ \\
\hline \multicolumn{2}{|l|}{$\boldsymbol{\sigma}^{2} \mathrm{gca} / \boldsymbol{\sigma}^{2} \mathrm{sca}$} & 0.30 & 0.94 & 0.22 & -0.029 & 0.82 & 0.84 & 0.52 & 0.05 & 0.66 & 0.22 \\
\hline
\end{tabular}

* and** indicates significant at $\mathrm{P}=0.05$ and $\mathrm{P}=0.01$ levels, respectively. 
Table.3 Estimation of general combining ability (gca) effects of parents for various characters in pearl millet

\begin{tabular}{|c|c|c|c|c|c|c|c|c|c|c|}
\hline PARENTS & $\begin{array}{c}\text { Days to } \\
\text { flowering }\end{array}$ & $\begin{array}{l}\text { Days to } \\
\text { maturity }\end{array}$ & $\begin{array}{l}\text { Plant } \\
\text { height }\end{array}$ & $\begin{array}{c}\text { No. of } \\
\text { effective } \\
\text { tillers } \\
\text { per plant }\end{array}$ & $\begin{array}{c}\text { Ear head } \\
\text { length }\end{array}$ & $\begin{array}{c}\text { Ear head } \\
\text { girth }\end{array}$ & $\begin{array}{c}\text { Test } \\
\text { weight }\end{array}$ & $\begin{array}{c}\text { Grain } \\
\text { yield per } \\
\text { plant }\end{array}$ & $\begin{array}{c}\text { Harvest } \\
\text { index }\end{array}$ & $\begin{array}{l}\text { Protein } \\
\text { content }\end{array}$ \\
\hline \multicolumn{11}{|l|}{ Lines } \\
\hline ICMA 06777 & $7.02 * *$ & $7.63 * *$ & $-19.31 * *$ & $0.27 * *$ & $-2.45 * *$ & $3.19 * *$ & -0.09 & $1.28 *$ & 0.84 & $-0.31 *$ \\
\hline ICMA 07777 & $1.96 * *$ & $4.17 * *$ & $-9.43 *$ & $0.17 * *$ & -0.21 & $2.59 * *$ & $0.38 * *$ & $1.52 *$ & $-3.17 * *$ & $-0.67 * *$ \\
\hline ICMA 96222 & $-1.77 * *$ & $-1.42 * *$ & $20.78 * *$ & $-0.52 * *$ & $3.27 * *$ & -0.32 & $1.36 * *$ & $3.46 * *$ & $10.99 * *$ & $0.27 *$ \\
\hline ICMA 97111 & $-3.63 * *$ & $-4.22 * *$ & $11.90 *$ & $0.31 * *$ & $-0.96 *$ & $-2.80 * *$ & $0.55 * *$ & $-2.81 * *$ & $5.30 * *$ & -0.22 \\
\hline ICMA 98444 & $-0.77 *$ & $-0.96 * *$ & -6.49 & $-0.27 * *$ & $-0.90 *$ & $0.85 *$ & $-0.32 * *$ & -0.53 & -0.84 & $0.55 * *$ \\
\hline ICMA 04999 & $-2.83 * *$ & $-5.16 * *$ & $-18.61 * *$ & -0.04 & $1.30 * *$ & $-6.81 * *$ & $-1.28 * *$ & $-1.74 * *$ & $-9.17 * *$ & $-0.38 * *$ \\
\hline ICMA 05444 & 0.02 & -0.02 & $21.17 * *$ & 0.06 & -0.04 & $3.29 * *$ & $-0.59 * *$ & -1.17 & $-3.94 * *$ & $0.77 * *$ \\
\hline S.Em. \pm & 0.64 & 0.50 & 9.41 & 0.08 & 0.87 & 0.78 & 0.21 & 1.27 & 1.43 & 0.25 \\
\hline \multicolumn{11}{|l|}{ Testers } \\
\hline $18488 \mathrm{R}$ & $0.75 * *$ & $2.23 * *$ & $23.54 * *$ & -0.01 & $4.35^{* *}$ & $0.98 * *$ & $1.04 * *$ & $2.32 * *$ & 1.11 & -0.21 \\
\hline $18587 \mathrm{R}$ & $2.08 * *$ & $2.09 * *$ & 7.18 & $0.10 * *$ & 0.42 & $1.54 * *$ & -0.04 & 0.29 & -0.00 & -0.02 \\
\hline $18805 \mathrm{R}$ & $-1.96 * *$ & $-1.47 * *$ & 7.28 & $0.12 * *$ & -0.63 & $2.47 * *$ & 0.06 & 0.59 & 0.72 & $0.46 * *$ \\
\hline $17369 \mathrm{R}$ & $-0.67 *$ & $-0.47 *$ & $-22.92 * *$ & $-0.23 * *$ & $-2.25 * *$ & $-2.34 * *$ & $-0.65 * *$ & $-1.81 * *$ & 0.67 & $-0.43 * *$ \\
\hline $17548 \mathrm{R}$ & -0.2 & $-2.38 * *$ & $-15.08 * *$ & 0.00 & $-1.89 * *$ & $-2.65 * *$ & $-0.41 * *$ & $-1.39 *$ & $-2.50 * *$ & 0.21 \\
\hline S.Em. \pm & 0.54 & 0.42 & 7.95 & 0.07 & 0.73 & 0.66 & 0.17 & 1.08 & 1.21 & 0.21 \\
\hline
\end{tabular}

* and ${ }^{* *}$ indicates significant at $\mathrm{P}=0.05$ and $\mathrm{P}=0.01$ levels, respectively. 
Table.4 Top three ranking parent with respect to per se performance and gca effects and three top ranking hybrids with respect to per se performance and sca effects and heterosis over better parent and standard check (GHB 558) in pearl millet.

\begin{tabular}{|c|c|c|c|c|c|c|c|c|c|c|}
\hline \multirow[b]{2}{*}{$\begin{array}{l}\text { Sr. } \\
\text { No. }\end{array}$} & \multirow[b]{2}{*}{ Characters } & \multirow{2}{*}{\begin{tabular}{|c|} 
Best \\
performing \\
parent (per se \\
performing)
\end{tabular}} & \multirow[b]{2}{*}{$\begin{array}{c}\text { Best } \\
\text { combiners }\end{array}$} & \multirow{2}{*}{$\begin{array}{c}\text { Best performing } \\
\text { hybrids } \\
\text { (per se } \text { performing) }\end{array}$} & \multirow{2}{*}{$\begin{array}{c}\text { Status } \\
\text { of } \\
\text { parents }\end{array}$} & \multirow[b]{2}{*}{$\begin{array}{l}\text { Hybrids with high sca } \\
\text { effects }\end{array}$} & \multirow{2}{*}{$\begin{array}{c}\text { GCA of } \\
\text { the } \\
\text { parents }\end{array}$} & \multirow[b]{2}{*}{$\begin{array}{c}\text { Sca } \\
\text { effects }\end{array}$} & \multicolumn{2}{|c|}{ Heterosis (\%) over } \\
\hline & & & & & & & & & $\begin{array}{l}\text { Better } \\
\text { parent }\end{array}$ & Standard check \\
\hline \multirow{3}{*}{1.} & \multirow{3}{*}{$\begin{array}{l}\text { Days to } \\
\text { flowering }\end{array}$} & ICMA 96222 & ICMA 97111 & ICMA $04999 \times 17369 \mathrm{R}$ & $\mathrm{G} \times \mathrm{G}$ & ICMA 07777 x $18805 \mathrm{R}$ & $P \times G$ & -5.77 & -17.61 & -1.36 \\
\hline & & ICMA 97111 & ICMA 04999 & ICMA $04999 \times 18805 \mathrm{R}$ & $\mathrm{G} \times \mathrm{G}$ & ICMA $05444 \times 17548 \mathrm{R}$ & $\mathrm{A} \times \mathrm{A}$ & -5.26 & -6.41 & -0.68 \\
\hline & & ICMA 07777 & $18805 \mathrm{R}$ & ICMA $97111 \times 17548 \mathrm{R}$ & $\mathrm{G} \times \mathrm{A}$ & ICMA 98444 x 17548 R & $\mathrm{G} \times \mathrm{A}$ & -5.13 & -11.11 & -2.04 \\
\hline \multirow{3}{*}{2.} & \multirow{3}{*}{$\begin{array}{l}\text { Days to } \\
\text { maturity }\end{array}$} & ICMA 96222 & ICMA 04999 & ICMA $04999 \times 18805$ & $\mathrm{G} \times \mathrm{G}$ & ICMA $07777 \times 18805 \mathrm{R}$ & $P \times G$ & -6.12 & -7.69 & -6.56 \\
\hline & & ICMA 06777 & ICMA 97111 & ICMA $04999 \times 17369$ & $\mathrm{G} \times \mathrm{G}$ & ICMA $97111 \times 18587 \mathrm{R}$ & $\mathrm{G} \times \mathrm{P}$ & -3.62 & -12.30 & -9.43 \\
\hline & & $17548 \mathrm{R}$ & $17548 \mathrm{R}$ & ICMA $04999 \times 17548$ & $G \times P$ & ICMA $05444 \times 17369 \mathrm{R}$ & $A \times G$ & -3.5 & -15.36 & -7.38 \\
\hline \multirow{3}{*}{3.} & \multirow{3}{*}{$\begin{array}{l}\text { Plant height } \\
\text { (cm) }\end{array}$} & ICMA 07777 & 17369 & ICMA 04999 x 17548 & $\mathrm{G} \times \mathrm{G}$ & ICMA 06777 x $18587 \mathrm{R}$ & $\mathrm{G} \times \mathrm{A}$ & -63.66 & -48.19 & -53.60 \\
\hline & & ICMA 06777 & ICMA 06777 & ICMA 06777 x 18587 & $\mathrm{G} \times \mathrm{A}$ & ICMA $04999 \times 17548 \mathrm{R}$ & Gx G & -60.89 & -58.31 & -63.51 \\
\hline & & ICMA 98444 & ICMA 04999 & ICMA 06777 x 17369 & $\mathrm{G} \times \mathrm{G}$ & ICMA $07777 \times 17369 \mathrm{R}$ & $\mathrm{G} \times \mathrm{G}$ & -38.43 & -50.65 & -50.97 \\
\hline \multirow{3}{*}{4.} & \multirow{3}{*}{$\begin{array}{l}\text { No. of } \\
\text { effective } \\
\text { tillers/plant }\end{array}$} & 18805 & ICMA 97111 & ICMA $97111 \times 18587$ & $G \times P$ & ICMA $97111 \times 18587 \mathrm{R}$ & $\mathrm{G} \times \mathrm{P}$ & 1.08 & 35.87 & 71.23 \\
\hline & & ICMA 07777 & ICMA 06777 & ICMA 07777x 18805 & $\mathrm{G} \times \mathrm{P}$ & ICMA 98444 x 17548 R & $\mathrm{P} \times \mathrm{A}$ & 0.94 & 7.69 & 15.07 \\
\hline & & 18488 & ICMA 07777 & ICMA 06777 x 17548 & $\mathrm{G} \times \mathrm{A}$ & ICMA $04999 \times 18488 \mathrm{R}$ & $\mathrm{A} \times \mathrm{A}$ & 0.70 & 3.12 & 35.62 \\
\hline \multirow{3}{*}{5.} & \multirow{3}{*}{$\begin{array}{l}\text { Ear head } \\
\text { length }(\mathrm{cm})\end{array}$} & 18488 & 18488 & ICMA 96222 x 18488 & $\mathrm{G} \times \mathrm{G}$ & ICMA 06777 x $18805 \mathrm{R}$ & $\mathrm{P} \times \mathrm{A}$ & 4.36 & 13.34 & 16.02 \\
\hline & & ICMA 07777 & ICMA 96222 & ICMA $04999 \times 18488$ & Gx G & ICMA $96222 \times 17369 \mathrm{R}$ & Gx P & 4.10 & 26.41 & 34.17 \\
\hline & & 17369 & ICMA 04999 & ICMA $96222 \times 18587$ & $\mathrm{G} \times \mathrm{A}$ & ICMA $98444 \times 17548 \mathrm{R}$ & $\mathrm{P} \times \mathrm{P}$ & 2.80 & 23.67 & 10.02 \\
\hline \multirow{3}{*}{6.} & \multirow{3}{*}{$\begin{array}{l}\text { Ear head } \\
\text { girth }(\mathbf{m m})\end{array}$} & ICMA 07777 & ICMA 05444 & ICMA 06777 x 18805 & $\mathrm{GxG}$ & ICMA 06777 x $17369 \mathrm{R}$ & $\mathrm{G} \times \mathrm{P}$ & 6.07 & 26.75 & 21.25 \\
\hline & & 18587 & ICMA 06777 & ICMA 05444 x 18488 & $\mathrm{G} \times \mathrm{G}$ & ICMA $04999 \times 18587 \mathrm{R}$ & $P \times G$ & 3.42 & -6.46 & -6.4 \\
\hline & & 18488 & ICMA 07777 & ICMA 07777 x 18805 & Gx G & ICMA $98444 \times 17548 \mathrm{R}$ & G x P & 3.29 & 13.07 & 4.1 \\
\hline \multirow{3}{*}{7.} & \multirow{3}{*}{$\begin{array}{l}\text { Test weight } \\
\text { (g) }\end{array}$} & ICMA 07777 & ICMA 96222 & ICMA 07777 x $18488 \mathrm{R}$ & $\mathrm{G} \times \mathrm{G}$ & ICMA 07777 x $18488 \mathrm{R}$ & $\mathrm{G} \times \mathrm{G}$ & 1.50 & 5.93 & 33.67 \\
\hline & & $17369 \mathrm{R}$ & $18488 \mathrm{R}$ & ICMA $96222 \times 18587 \mathrm{R}$ & $\mathrm{G} \times \mathrm{A}$ & ICMA 06777 x $18805 \mathrm{R}$ & $\mathrm{A} \times \mathrm{A}$ & 1.32 & 5.85 & 17.76 \\
\hline & & $18488 \mathrm{R}$ & ICMA 97111 & ICMA 96222 x $18488 \mathrm{R}$ & $\mathrm{G} \times \mathrm{G}$ & ICMA 96222 x $18587 \mathrm{R}$ & $\mathrm{G} \times \mathrm{A}$ & 1.08 & 15.77 & 28.48 \\
\hline \multirow{3}{*}{8.} & \multirow{3}{*}{$\begin{array}{l}\text { Grain yield/ } \\
\text { plant } \\
\text { (g) }\end{array}$} & $17548 \mathrm{R}$ & ICMA 96222 & ICMA 07777 x $18488 \mathrm{R}$ & $\mathrm{G} \times \mathrm{G}$ & ICMA 07777 x $18488 \mathrm{R}$ & $\mathrm{G} \times \mathrm{G}$ & $9.53 * *$ & $52.60 * *$ & $32.56^{* * *}$ \\
\hline & & ICMA 96222 & $18488 \mathrm{R}$ & ICMA 06777 x $18805 \mathrm{R}$ & $\mathrm{G} \times \mathrm{A}$ & ICMA 06777 x $18805 \mathrm{R}$ & $\mathrm{G} \times \mathrm{A}$ & $9.17 * *$ & $52.12 * *$ & $25.78^{* *}$ \\
\hline & & ICMA 98444 & ICMA07777 & ICMA $96222 \times 18488 \mathrm{R}$ & $\mathrm{G} \times \mathrm{G}$ & ICMA $96222 \times 18587 \mathrm{R}$ & $\mathrm{G} \times \mathrm{A}$ & $5.42 * *$ & $25.65 * *$ & $20.34 * *$ \\
\hline \multirow{3}{*}{9.} & \multirow{3}{*}{$\begin{array}{l}\text { Harvest } \\
\text { Index }(\%)\end{array}$} & $17548 \mathrm{R}$ & ICMA 96222 & ICMA $96222 \times 17369 \mathrm{R}$ & $\mathrm{G} \times \mathrm{A}$ & ICMA $04999 \times 17548 \mathrm{R}$ & $\mathrm{P} \times \mathrm{P}$ & 7.17 & -23.85 & -17.13 \\
\hline & & ICMA 96222 & ICMA 07777 & ICMA $96222 \times 17548 \mathrm{R}$ & $\mathrm{G} \times \mathrm{P}$ & ICMA 07777 x 17548 R & $\mathrm{P} \times \mathrm{P}$ & 6.70 & -19.28 & -12.17 \\
\hline & & ICMA 98444 & ICMA 06777 & ICMA 96222 x $18488 \mathrm{R}$ & $\mathrm{G} \times \mathrm{A}$ & ICMA 98444 x 18805 R & $\mathrm{A} \times \mathrm{A}$ & 6.05 & -5.07 & -2.88 \\
\hline & & $17548 \mathrm{R}$ & ICMA 05444 & ICMA 05444 x $18587 \mathrm{R}$ & $\mathrm{G} \times \mathrm{A}$ & ICMA $97111 \times 18805 \mathrm{R}$ & $\mathrm{A} \times \mathrm{G}$ & 1.58 & 15.79 & 22.99 \\
\hline 10. & $\begin{array}{l}\text { Proten } \\
\text { content }(\%)\end{array}$ & ICMA 05444 & ICMA 98444 & ICMA 98444 x $17369 \mathrm{R}$ & Gx P & ICMA 05444 x $18805 \mathrm{R}$ & Gx $\mathrm{x}$ & 1.54 & 14.32 & 34.46 \\
\hline & & ICMA 18587 & $18805 \mathrm{R}$ & ICMA $97111 \times 18587 \mathrm{R}$ & $\mathrm{A} \times \mathrm{A}$ & ICMA $98444 \times 17548 \mathrm{R}$ & $\mathrm{G} \times \mathrm{A}$ & 1.46 & -2.43 & 27.92 \\
\hline
\end{tabular}
*, ** Significant at $\mathrm{P}=0.05$ and $\mathrm{P}=0.01$ levels respectively. 
The mean squares due to males were significant for days to maturity, ear head length, ear head girth, test weight and grain yield per plant. This indicated the importance of males for their contribution towards general combining ability variance components. The line $\times$ tester mean squares exhibited significance for all plant traits. The ratio of $\sigma_{\text {gca }}^{2} / \sigma_{\text {sca }}^{2}$ was less than unity for all the characters under study (Table 2). This suggested greater role of non-additive genetic variance in the inheritance of all the traits under study. Among parents, ICMA 07777, ICMA 96222 and 18488 R were good general combiner for grain yield per plant, plant height, number of effective tillers per plant. The female ICMA 96222 was found as good general combiner for characters like grain yield per plant, days to flowering, days to maturity, test weight, harvest index and protein content. While ICMA 07777 was good combiner for days to flowering, ear head girth and test weight and harvest index. Among males, 18488-R was found as a good combiner for grain yield per plant, ear head length, ear head girth and test weight (Table 3). This suggested that parents might be presumed to have relatively greater number of favorable alleles for developing superior hybrids/varieties of pearl millet (Table 4). Out of 35 hybrids, three hybrids viz., ICMA 07777 $\times 18488 \mathrm{R}(9.53)$, ICMA $06777 \times 18805 \mathrm{R}$ (9.17) and ICMA $96222 \times 18587 \mathrm{R}(5.42)$ exhibited significant positive specific combining ability effects for grain yield per plant. Earlier significant GCA and SCA effects in pear millet for grain yield and component traits also reported by Jagendra Singh et al., (2014), Patel et al., (2014), Khandagale et al., (2014), Priyanka (2015) and Mungra et al., (2015).

With regard to heterosis over mid parent, better parent and standard heterosis, exhibited high heterosis in desired direction for all the characters. The hybrids viz., ICMA $07777 \mathrm{x}$
18488 R, ICMA 06777 x 18805 R and ICMA $96222 \times 18488 \mathrm{R}$ showed high per se performance with high significant positive heterobeltiosis standard heterosis for grain yield per plant (Table 4). The results of heterosis were in accordance with the studies conducted by of Yadav (2006) and Pawar et $a l$. , (2015) for days to flowering. For days to maturity, reported by Yadav et al., (2000) and Manga et al., (2004). For ear head length and ear head girth was in accordance with the studies of, Manga et al., (2004) and Vetriventhan et al., (2008). For grain yield, reported by Bachkar et al., (2014), Bhuri singh et al., (2015) and Priyanka (2015).

In general all characters, the cross showing positive and negative combining ability effect, involved either good $\times$ good, average $\times$ good, good $\times$ poor, average $\times$ average, average $\times$ poor and poor $\times$ poor general combiners. Crosses between good $\times$ poor and poor $\times$ good combiners could be attributed to lack of co-adaptation between favourable alleles of the parents involved. Generally the cross showing high sca effects for grain yield per plant also exhibited high or average or poor sca effects for yield components (Table 4). This indicated that a yield is a complex characters depending upon number of component characters.

The cross ICMA $07777 \times 18488 \mathrm{R}$ showed high per se performance, significant heterobeltiosis, standard heterosis and significant positive specific combining ability effect and high sca effects involving one good general combiner, indicated additive $\times$ dominance type of interaction, which can produce desirable transgressive segregate in subsequent generations, also used for exploiting commercial cultivation. The cross ICMA $06777 \times 18805$ had good $\times$ average combiner parents, high per se performance, significant positive heterobeltiosis and standard heterosis and positive specific 
combining ability effect for grain yield per plant, test weight, ear head length and harvest index. The cross ICMA $96222 \times 18488 \mathrm{R}$ had good $\times$ good combiner parent, significant positive specific combining effect for grain yield per plant test weight, ear head length and protein content. This indicated to obtain desirable transgressive sergeants in $F_{2}$ or subsequent generation, may be used for development of pollinated CMS line as female parent and pollen fertility restorer line as male parent for future breeding programme of grain yield per plant, production of high yielding hybrids and for commercial purpose.

\section{References}

Athwal, D. S. (1965). Hybrid Bajra-1 marks a new era. Indian Farming., 15: 6-7.

Bachkar, R. M., Pole, S. P and Patil, S. N. 2014. Heterosis for Grain Yield and Its Components in Pearl Millet (Pennisetum glaucum L.). Indian Journal of Dryland Agricultural Research and Development. 29 (1): 4044.

Bhuri Singh, Sharma, K. C., Mittal, G. K and Meena, H. K. 2015. Heterosis for grain yield and its component traits in pearl millet in different environments. International Journal of Tropical Agriculture. 33(1): 47-51.

Briggle, L.W. 1963. Heterosis in Wheat - A review. Crop Sci., 3(3): 407-412.

Burton, G. W. 1958. Cytoplasmic male sterility in pearl millet (Pennisetum glaucum (L.) R. Br.). Agronomy Journal, 50 (2): 230-231.

Fonesca, S. and Patterson, F. L. 1968. Hybrid vigour in a seven parents diallel cross in common winter wheat ( $T$. aestivum L.). Crop Science., 8: 85-88.

Hanna, W. W. 1989. Characteristics and stability of a new cytoplasmic-nuclear male-sterile source in pearl millet. Crop Science, 29 (4): 1457-1459.
Jagendra Singh and Ravi Sharma 2014. Assessment of Combining Ability in pearl millet Using Line $\mathrm{x}$ Tester Analysis. Advances in Crop Science and Technology. 2: 4.

Kempthorne, O. 1957. An Introduction to Genetic Statistics. John Willey \& Sons. Inc., New York. : 545.

Khandagale, S. G., Vijay Sharma; Lone, R. P., Khandagale, V. G and Kumara Swamy, R.V. 2014. Combining Ability analysis and gene action in pearl millet [Pennisetum glaucum (L.) R. Br.]. Electronic Journal of Plant Breeding. 5 (3): 445-450.

Manga, V. K., Khan, A. K. F and Dubey, L. K. 2004. Identification of suitable inbreds based on combining ability in pearl millet [Pennisetum glaucum (L.) R. BR]. Indian Journal of Agricultural Science. 74 (2): 98-101.

Meredith, W.R. and Bridge, R.R. 1972. Heterosis and gene action in cotton Gossypium hirsutum. Crop Science. 12 : 304-310.

Mungra, K. S., Dobariya, K. L., Sapovadiya, M. H and Vavdiya, P. A. 2015. Combining ability and gene action for grain yield and its component traits in pearl millet [Pennisetum glaucum (L.) R. Br.]. Electronic Journal of Plant Breeding. 6 (1): 66-73.

Patel, S. M., Patel, M. P., and Patel.J. A. 2014. Combining ability and gene action for grain yield and agronomic traits in pearl millet restorer lines. Electronic Journal of Plant Breeding. 5 (3): 395-401.

Pawar, V. Y., Kute, N. S., Patil, H.T., Awari, V.R., Gavali, R. K. and Deshmukh, G. P. 2015. Heterosis for earliness in pearl millet [Pennisetum glaucum (L.) R. Br.]. Bioinfolet. 12(3B): 696-706.

Priyanka, 2015. Heterosis and combining ability analysis for seed yield and its quality traits in pearl millet[Pennisetum 
glaucum (L.) R. Br.]. M.Sc. (Agri.) Thesis (unpublished) submitted to SDAU, Sardarkrushinagar.

Rai K. N., Anand Kumar, K., Andrews, D. J. and Rao A. S. 2006. Commercial viability of alternative cytoplasmicnuclear male-sterility systems in pearl millet. Euphytica, 121 (3): 107-114.

Sukhatme, P.V. and Amble, V.N. 1989. Statistical Methods for Agricultural Workers, ICAR, New Delhi.

Vetriventhan, M., Nirmalakumari, A. and Ganapathy, S. 2008. Heterosis for Grain Yield Components in Pearl Millet
(Pennisetum glaucum (L.) R. Br.). World Journal of Agricultural Sciences 4 (5): 657-660.

Yadav, O. P. 2006. Heterosis in crosses between landraces and elite exotic populations of pearl millet [Pennisetum glaucum (L.) R. Br.] in arid zone environments. Indian Journal of Genetics, 66 (4): 308-311.

Yadav, O. P., Weltzein, E. R., Bidinger, F. R. and Mahalakshmi, V. 2000. Heterosis in land race based on top cross hybrids of pearl millet across arid environments. Euphytica, 112 (3): 285-295.

\section{How to cite this article:}

Reshma Krishnan, M.R., M.S. Patel, R.A. Gami, H.S. Bhadauria and Patel, Y.N. 2017. Genetic Analysis in Pearl Millet [Pennisetum glaucum (L) R. Br.]. Int.J.Curr.Microbiol.App.Sci. 6(11): 900-907. doi: https://doi.org/10.20546/ijcmas.2017.611.105 